\title{
Evaluation of humoral pattern detection performance of Mindray BC-6000PLUS blood analyzer based on WS/T 662-2020
}

\author{
Yifei Long ${ }^{1}$, Jianping Liu ${ }^{1}$, Fu Chen ${ }^{1}$, Yanfen Luo ${ }^{1}$, Chanjing Zhao ${ }^{1}$, Jianyang Wei ${ }^{2}$, Cha Chen ${ }^{1}$, \\ Dongling Lin ${ }^{1}$
}

${ }^{1}$ Department of Laboratory, Guangdong Hospital of Traditional Chinese Medicine, Second Affiliated Hospital of Guangzhou University of Chinese Medicine, Guangzhou, China; ${ }^{2}$ Shenzhen Mindray Bio-Medical Electronic Co., Ltd., Shenzhen, China

Contributions: (I) Conception and design: Y Long, D Lin; (II) Administrative support: D Lin; (III) Provision of study materials or patients: J Liu, F Chen; (IV) Collection and assembly of data: Y Long, C Zhao, J Wei, C Chen; (V) Data analysis and interpretation: Y Long, D Lin; (VI) Manuscript writing: All authors; (VII) Final approval of manuscript: All authors.

Correspondence to: Dongling Lin. Department of Laboratory, Guangdong Hospital of Traditional Chinese Medicine, Second Affiliated Hospital of Guangzhou University of Chinese Medicine, Guangzhou 510006, China. Email: 1d113929547161@126.com.

\begin{abstract}
Background: Manual microscopic examination is the gold standard of humoral cell count test. However, it has some limitations and cannot fully meet clinical needs. Compared with the manual method, the automatic blood cell analyzer has the advantages of a high degree of automation, minimal error, high speed, high precision, and easy standardization. This study intends to verify the detection performance of the body fluid model of the Mindray BC-6000PLUS automatic hematology analyzer.

Methods: This study was performed in accordance with the International Committee for Standardization in Haematology (ICSH) Hematology Analyzer Evaluation Guide (version 2014) and the requirements of WS/T662-2020 "Clinical humoral examination technique". The humoral white blood cell-body fluid (WBC-BF), humoral red blood cell-body fluid (RBC-BF), monocyte (MN), polymorphonuclear (PMN) were measured to verify the performance indicators of the instrument, including background counting, intrabatch precision, accuracy, carrying contamination rate, and linear range. Referring to the WS/T514-2017 (Establishment and verification of detection capability for clinical laboratory measurement procedures), the limit of blank $(\mathrm{LoB})$ and limit of detection $(\mathrm{LoD})$ values of WBC-BF and RBC-BF in the humoral mode of the instrument were established.
\end{abstract}

Results: The blank count of WBC-BF and RBC-BF, and contamination of Mindray BC-6000PLUS analyzer were zero; the coefficient of variation $(\mathrm{CV})$ of intra-batch precision at different levels of each item was less than $10 \%$. There was a high correlation between instrument test results and manual microscopic examination results $(r>0.95)$. The linear range of the instrument was wide, and the linear verification parameters was good $\left(\mathrm{R}^{2}>0.999\right)$. The $\mathrm{LoB}$ value and $\mathrm{LoD}$ value of $\mathrm{WBC}-\mathrm{BF}$ established by the instrument were $0 \times 10^{9} / \mathrm{L}$ and $0.004 \times 10^{9} / \mathrm{L}$, respectively. The $\mathrm{LoB}$ value and $\mathrm{LoD}$ value of the $\mathrm{RBC}-\mathrm{BF}$ established by the instrument were $0 \times 10^{12} / \mathrm{L}$ and $0.004 \times 10^{12} / \mathrm{L}$, respectively. The lower detection limits of WBC-BF and RBC-BF were set as $0.004 \times 10^{9} / \mathrm{L}$ and $0.004 \times 10^{12} / \mathrm{L}$, respectively.

Conclusions: All performance indicators of the Mindray BC-6000PLUS automatic blood analyzer met the requirements of the manufacturer's criteria. This instrument can fulfill the requirement of body fluid sample routine test in clinical practice.

Keywords: WS/T662-2020; humoral mode; performance evaluation (PE)

Submitted Mar 20, 2021. Accepted for publication Jun 11, 2021.

doi: 10.21037/apm-21-1187

View this article at: https://dx.doi.org/10.21037/apm-21-1187 


\section{Introduction}

In clinic practice, cerebrospinal fluid, serous cavity effusion, and articular cavity effusion are common and important humoral samples of laboratory tests. The routine test of these samples, especially cell count, has great significances for differential diagnosis, treatment, assessment of disease progression, and prognosis of patients (1). Manual microscopic examination and classification of body fluid samples has become the gold standard of cytological examination for a long time (2). However, it has some drawbacks, such as the high subjectivity on cell discrimination, high requirements of experience and technology, poor repeatability, low precision, and time-consuming operation. With the development of flow cytometry in recent years, many automatic blood cell analyzers have been developed to perform the functions of cell counting and classification for analyzing body fluid samples. These instruments have a high degree of automation and less manual operation-related errors, which improved the detection efficiency, accuracy and precision, and facilitated the procedure of standardization. Given their high application value, cell counting and cell classification of body fluid samples are being more widely processed by automatic hematology analyzers (3-5). The Mindray BC-6000PLUS automatic hematology analyzer (hereinafter referred to as the BC-6000PLUS analyzer; Shenzhen Mindray Biomedical Electronics Co., Ltd., Shenzhen, China) has 2 detection modes (i.e., blood model and humoral mode). The humoral mode can not only count white blood cell-body fluid (WBC-BF) and red blood cell-body fluid (RBC-BF), but can also provide research data such as classified counting of nucleated cells and high fluorescence intensity cells. This study intended to verify the performance of the humoral mode of the BC-6000PLUS analyzer according to the criteria of the current guidelines, and establish the limit of blank (LoB) and limit of detection (LoD) of the cell count in order to evaluate and understand the feasibility of the BC6000PLUS analyzer's humoral mode in clinical application. Based on this, we determined the lower detection limits of WBC-BF and RBC-BF. We present the following article in accordance with the MDAR reporting checklist (available at https://dx.doi.org/10.21037/apm-21-1187).

\section{Methods}

\section{Study design}

This is a retrospective study. All procedures performed in this study involving human participants were in accordance with the Declaration of Helsinki (as revised in 2013). It only collects the clinical data of patients, does not interfere with the treatment plan of patients, and will not bring physiological risks to patients. Researchers will try their best to protect the information provided by patients, do not disclose personal privacy, and exempt informed consent.

\section{Specimen sources}

For specimen collection, 55 humoral samples (23 cerebrospinal fluid and 32 hydrothorax and ascites) from inpatients at the University City Hospital of Guangdong Hospital of Traditional Chinese Medicine in May 2020 were collected and mixed in EDTA-2K anticoagulant tubes and then sent for examination. The samples were counted and classified within 1 hour according to WS/T662-2020 "Clinical Humoral examination technology."

\section{Instruments and reagents}

The following instruments and reagents were acquired: a Mindray BC-6000PLUS automatic blood cell analyzer and its supporting reagents (Shenzhen Mindray Biomedical Electronics Co., Ltd., Shenzhen, China), an Olympus CX-21 binocular microscope (Zeshi Optoelectronics Technology Co., Ltd., Shanghai, China), and a disposable FAST-READ10 urine sediment counting board (Shanghai Sango Biotechnology Co., Ltd., Shanghai, China).

\section{Study methods}

The performance parameters of the BC6000PLUS analyzer were verified by referring to the performance evaluation guidelines of the International Committee for Standardization in Haematology (ICSH) humoral cell automatic counter of 2014 and $(6,7)$ the requirements of WS/T662-2020 "Clinical humoral examination technique". The humoral white blood cell-body fluid (WBC-BF), humoral red blood cellbody fluid (RBC-BF), monocyte (MN), polymorphonuclear $(\mathrm{PMN})$ were measured to verify the performance indicators of the instrument, including background counting, intrabatch precision, accuracy, carrying contamination rate, and linear range. The $\mathrm{LoB}$ value and $\mathrm{LoD}$ value of $\mathrm{WBC}-\mathrm{BF}$ and RBC-BF were established by referring to the WS/T5142017 (Establishment and verification of detection capability for clinical laboratory measurement procedures). All the verification operation procedures were carried out in strict accordance with the instrument instructions and the fourth 
version of the National Operating Rules for Clinical Testing. In addition, the result judgment criteria were mainly based on the technical indicators provided by the manufacturers.

\section{Background count}

The BC-6000PLUS diluent (Shenzhen Mindray Biomedical Electronics Co., Ltd.) was used as a sample to be measured continuously 3 times in the body fluid mode of the analyzer under the normal state of the instrument. In addition, the maximum values of white blood cell (WBC) and red blood cell (RBC) counts were each taken. The maximum value was required to be within the allowable range of background detection set by the manufacturer.

\section{Contamination rate}

One clinical sample with high concentration and one with low concentration (the results were close to the linear high and low concentration) were mixed uniformly. Thereafter, the high concentration samples were detected consecutively 3 times (the test results were recorded as $\mathrm{H} 1, \mathrm{H} 2$, and $\mathrm{H} 3$ ). The low concentration sample was then tested consecutively for 3 times as well (the test results were recorded as L1, L2, and L3). The carrying pollution rate of $\mathrm{WBC}-\mathrm{BF}$ and RBC-BF (\%) were calculated with the following formula: carrying pollution rate $(\%)=(\mathrm{L} 1-\mathrm{L} 3) /(\mathrm{H} 3-\mathrm{L} 3) \times 100 \%$.

\section{Intra-batch precision}

One high concentration level sample and one low concentration level sample were tested 11 times repeatedly on the same day according to the conventional method (at least 5 times if the specimen was limited). The last 10 test results were selected to calculate the mean, standard deviation $(\mathrm{SD})$, and coefficient of variation $(\mathrm{CV})$ of $\mathrm{WBC}$ $\mathrm{BF}$ and RBC-BF.

\section{Accuracy}

The 55 clinical fluid samples were collected, divided into 2 samples, and examined by the BC-6000PLUS analyzer and manual microscope respectively within 1 hour (8). Operation of the manual mode was done in strict accordance with the fourth version of the National Rules of Clinical Examination. Moreover, 2 competent examiners with many years of clinical examination experience counted RBCs and WBCs, with the average of their counts being taken as the final counting results. The samples were centrifuged and stained with Wright's-Giemsa Staining Solution (GBCBIO Technologies) for leukocyte count. Finally, the correlation between the results of the BC-
6000PLUS analyzer including the counting of the WBC, $\mathrm{RBCs}$, mononuclear cells (MNs, including monocytemacrophage-like cells, lymphocytes mesothelial cells, etc.), and multiple nuclear cells (PMNs, including neutrophils, eosinophils, and basophils) and those of the manual mode were analyzed.

\section{Linear range verification}

One cerebrospinal fluid sample whose WBC and RBC were close to the upper LoD (it is not recommended to take the hydrothorax and ascites sample as the linear verification sample because the cells in the high value hydrothorax and ascites sample are easy to gather and adhere) was diluted by the supporting diluent of the instrument with the proportions of $1: 2,1: 4,1: 8,1: 16,1: 32,1: 64,1: 128,1: 512$, and 1:1,024. Then, each dilution sample was measured 3 times, and the mean value was calculated under the humoral mode. The measured values were compared with the theoretical values $(\mathrm{Y}=\mathrm{ax}+\mathrm{b})$, and the measured mean values and theoretical values were analyzed by linear regression analysis.

\section{Establishment of the LoB and LoD}

The $\mathrm{LoB}$ and $\mathrm{LoD}$ was established according to the WS/ T514-2017 "Establishment and verification of detection ability of clinical testing methods" (9).

The LoB refers to the highest measured value obtained from multiple blank specimens after repeated measurement. BC-6000PLUS diluent was used as the test sample and was repeatedly tested 10 times a day for 6 days under the humoral mode of the analyzer, yielding 60 data sets. The LoD was determined by low-concentration samples. Six low concentration samples (the optimal concentration range from LoB to 4 times LoB) were tested 10 times in different time periods within 1 day, and 60 data sets were obtained.

\section{Statistical analysis}

The data were analyzed by using SPSS 20.0 (IBM Corp.) or Excel 2010 (Microsoft Corp.) software where appropriate. The nonparametric tests were used to analyze the data with nonnormal distribution.

\section{Results}

\section{Background count}

The diluent was adopted as the sample, and the WBC and $\mathrm{RBC}$ counts were determined 3 times in succession. The results 
Table 1 Background detection results of the BC-6000PLUS analyzer

\begin{tabular}{lcc}
\hline Parameters & WBC-BF $\left(10^{9} / \mathrm{L}\right)$ & RBC-BF $\left(10^{12} / \mathrm{L}\right)$ \\
\hline Max & 0.000 & 0.000 \\
Request & $\leq 0.001$ & $\leq 0.003$ \\
Conclusion & Qualified & Qualified \\
\hline
\end{tabular}

WBC-BF, white blood cell-body fluid; RBC-BF, red blood cell-body fluid.

Table 2 Carrying pollution rate of body the fluid model of the BC-6000PLUS analyzer

\begin{tabular}{lccccccccc}
\hline Item & $\mathrm{H} 1$ & $\mathrm{H} 2$ & $\mathrm{H} 3$ & $\mathrm{~L} 1$ & $\mathrm{~L} 2$ & $\mathrm{~L} 3$ & Carrying pollution rate\% & Request & Conclusion \\
\hline WBC-BF $\left(\times 10^{9}\right)$ & 10.364 & 10.074 & 10.267 & 0.007 & 0.010 & 0.007 & 0.00 & $\leq 0.30 \%$ \\
RBC-BF $\left(\times 10^{12}\right)$ & 4.701 & 4.711 & 4.558 & 0.005 & 0.005 & 0.005 & 0.00 & Qualified \\
\hline
\end{tabular}

WBC-BF, white blood cell-body fluid; RBC-BF, red blood cell-body fluid.

Table 3 Intra-batch precision results of the body fluid model of the BC-6000PLUS analyzer

\begin{tabular}{|c|c|c|c|c|}
\hline Parameters & \multicolumn{2}{|c|}{ Medium concentration level } & \multicolumn{2}{|c|}{ Low concentration level } \\
\hline Mean & 0.260 & 0.060 & 0.010 & 0.040 \\
\hline Standard deviation & 0.007 & 0.003 & 0.001 & 0.002 \\
\hline $\mathrm{CV}$ & $2.52 \%$ & $4.97 \%$ & $7.50 \%$ & $4.65 \%$ \\
\hline Conclusion & Qualified & Qualified & Qualified & Qualified \\
\hline
\end{tabular}

WBC, white blood cell; RBC, red blood cell; CV, coefficient of variation.

were all 0 , which met the background requirements of the manufacturers. The results are detailed in Table 1 (the allowable ranges set by the instrument manufacturers were WBC-BF $\leq 0.001 \times 10^{9} / \mathrm{L}$ and $\mathrm{RBC}-\mathrm{BF} \leq 0.003 \times 10^{12} / \mathrm{L}$, respectively).

\section{Carrying contamination rate}

The contamination rate of $\mathrm{WBC}-\mathrm{BF}$ and $\mathrm{RBC}-\mathrm{BF}$ measured in BC-6000PLUS humoral mode was $0 \%$, which met the pollution rate requirements of manufacturers $(\leq 0.3 \%)$. The determination results of high and low concentration samples are shown in Table 2.

\section{Intra-batch precision}

To determine intra-batch precision, the $\mathrm{WBC}-\mathrm{BF}$ and $\mathrm{RBC}-\mathrm{BF}$ values of medium and low concentration humoral samples were measured continuously 11 times under the humoral mode. The results of the 10 most recent measures were statistically analyzed, which revealed that all of the $\mathrm{CV}$ values were lower than the standard set by the instrument's manufacturer. The detailed results are shown in Table 3.

\section{Accuracy}

The correlations between the results of WBC-BF, RBC$\mathrm{BF}$, mononuclear cell $\mathrm{MN}$, and multiple nuclear cell $\mathrm{PMN}$ of 55 clinical samples detected by BC-6000PLUS and those of the manual microscopic examination were analyzed by linear regression. The results indicated that the WBC-BF, RBC-BF, MN, and PMN items of the 2 detection methods were highly correlated. The results of the correlation analysis were shown in Table 4 and the corresponding correlation diagrams were showed in Figure 1.

\section{Linear range}

In the linear analysis of $\mathrm{WBC}-\mathrm{BF}$ and $\mathrm{RBC}-\mathrm{BF}$, the linear range of $\mathrm{WBC}-\mathrm{BF}$ was $(0.008-10.235) \times 10^{9} / \mathrm{L}$; meanwhile, the linear regression equation was the following: $y=1.0032 x+0.025$ (with $\alpha=1.0032$ and $\mathrm{R}^{2}=0.9998$ ). 
Table 4 Correlation between the results of the BC-6000PLUS instrument method and manual method

\begin{tabular}{lcccc}
\hline Item & Regression equation & $\mathrm{R}^{2}$ & Criteria: correlation coefficient or slope & Conclusion \\
\hline WBC-BF & $\mathrm{y}=1.013 \mathrm{x}-4.7886$ & 0.9896 & $\mathrm{R} \geq 0.9$ and slope 0.7-1.3 & Qualified \\
RBC-BF & $\mathrm{y}=0.9143 \mathrm{x}+0.0245$ & 0.9917 & $\mathrm{R} \geq 0.8$ and slope 0.7-1.3 & Qualified \\
MN & $\mathrm{y}=0.9263 \mathrm{x}+2.5924$ & 0.9761 & $\mathrm{R} \geq 0.7$ & Qualified \\
PMN & $\mathrm{y}=1.118 \mathrm{x}-6.1496$ & 0.9743 & $\mathrm{R} \geq 0.7$ & Qualified \\
\hline
\end{tabular}

WBC-BF, white blood cell-body fluid; RBC-BF, red blood cell-body fluid; MN, monocyte; PMN, polymorphonuclear.

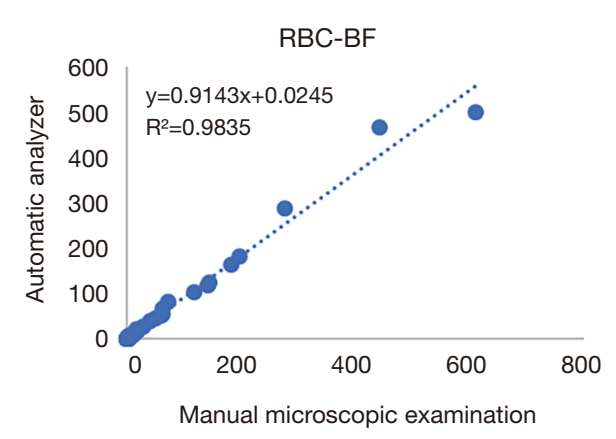

MN

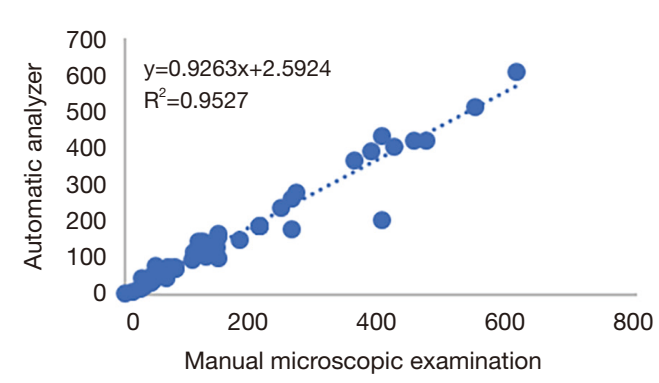

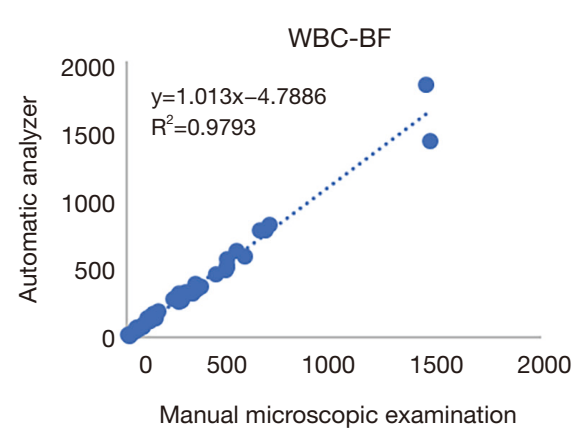

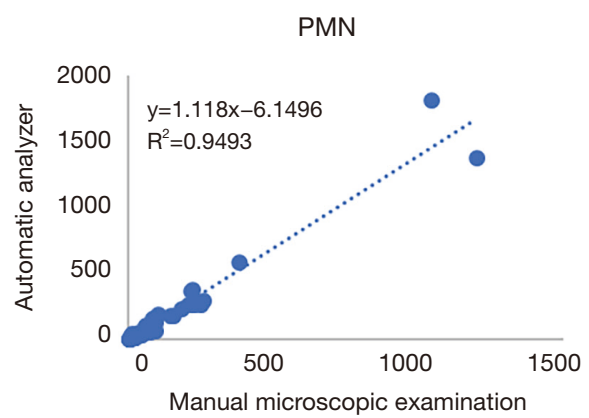

Figure 1 The correlation diagrams of the results of automatic analyzer and manual microscopic examination. WBC-BF, white blood cellbody fluid; RBC-BF, red blood cell-body fluid; MN, monocyte; PMN, polymorphonuclear.

In addition, the linear range of $\mathrm{RBC}-\mathrm{BF}$ was $(0.005-$ $4.657) \times 10^{12} / \mathrm{L}$; meanwhile the linear regression equation was the following: $y=0.9984 x-0.0021$ (with $\alpha=1.0032$ and $\left.\mathrm{R}^{2}=0.9998\right)$. This indicated that the linear range of the instrument's WBC-BF and RBC-BF was wide and was in line with the requirements of the instrument's manufacturer. The detailed results and diagrams of the linear analysis of WBC-BF and RBC-BF are shown in Table 5 and Figure 2.

\section{Establishment of the LoB and LoD for WBC-BF and RBC-BF}

For the LOB, 10 blank samples (the supporting diluent) were tested each day, and a total of 60 data sets were obtained after 6 days. The results showed that the detection values of $\mathrm{WBC}-\mathrm{BF}$ and $\mathrm{RBC}-\mathrm{BF}$ were 0 . Thus, for the humoral mode of our BC-6000PLUS analyzer, the LoB value of $\mathrm{WBC}-\mathrm{BF}$ was $0 \times 10^{9} / \mathrm{L}$, while the $\mathrm{LoB}$ value of $\mathrm{RBC}-\mathrm{BF}$ was $0 \times 10^{12} / \mathrm{L}$.

In determining the LoD, only 6 low-concentration samples were analyzed in different time periods within 1 day due to the limited stability of the humoral samples. The detection results of the samples are detailed in Tables 6 and 7 . After statistical analysis, the test results did not conform to a normal distribution. As a consequence, the $\mathrm{LoD}$ was established by using the nonparametric analysis method. The statistical formula was $\mathrm{LoD}=\mathrm{LoB}+\mathrm{D}_{\mathrm{s} \beta}$, where $\mathrm{D}_{\mathrm{s} \beta}$ was the interval between 
Table 5 Results of WBC-BF $\left(\times 10^{9} / \mathrm{L}\right)$ linear sample analysis

\begin{tabular}{lcccc}
\hline Item & Regression equation & $\mathrm{R}^{2}$ & Linear range & Assessment standard \\
\hline WBC-BF & $\mathrm{y}=1.0032 \mathrm{x}+0.025$ & 0.9998 & $0.008-10.235 \times 10^{9} / \mathrm{L}$ & $\mathrm{R}^{2} \geq 0.95$ and slope $0.95-1.05$ \\
RBC-BF & $\mathrm{y}=0.9984 x-0.0021$ & 0.9999 & $0.005-4.657 \times 10^{12} / \mathrm{L}$ & $\mathrm{R}^{2} \geq 0.95$ and slope $0.95-1.05$ \\
\hline
\end{tabular}

WBC-BF, white blood cell-body fluid; RBC-BF, red blood cell-body fluid.
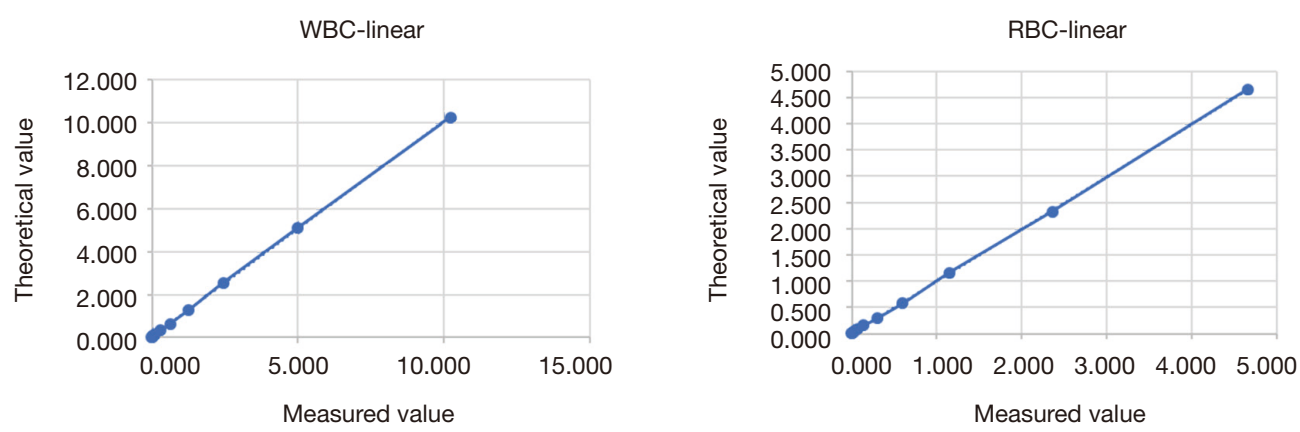

Figure 2 The linear regression analysis diagrams of the measured values and theoretical values. WBC, white blood cell; RBC, red blood cell.

Table 6 Detection results of low-concentration WBC-BF samples

\begin{tabular}{|c|c|c|c|c|c|c|}
\hline $\begin{array}{l}\text { Theoretical } \\
\text { concentration }\left(\times 10^{9} / \mathrm{L}\right)\end{array}$ & 0.001 & 0.002 & 0.004 & 0.006 & 0.008 & 0.010 \\
\hline 1 & 0.001 & 0.001 & 0.004 & 0.006 & 0.006 & 0.010 \\
\hline 3 & 0.001 & 0.002 & 0.004 & 0.006 & 0.008 & 0.013 \\
\hline 6 & 0.000 & 0.003 & 0.003 & 0.006 & 0.008 & 0.009 \\
\hline 7 & 0.002 & 0.003 & 0.002 & 0.005 & 0.007 & 0.010 \\
\hline 8 & 0.000 & 0.005 & 0.004 & 0.005 & 0.007 & 0.013 \\
\hline
\end{tabular}

WBC-BF, white blood cell-body fluid.

the median $(M)$ and the 5 th percentile of the test results.

The median LoD of WBC-BF was calculated by the following formula: $(M)=60 \times 0.5+0.5=$ the value of the $30.5 \mathrm{rank}=0.005 \times 10^{9} / \mathrm{L}$; meanwhile, the 5 th percentile was calculated by the formula as following: 5 th percentile $=60 \times 0.05+0.5=$ the value of the $3.5 \mathrm{rank}=0.001 \times 10^{9} / \mathrm{L}$. $\mathrm{D}_{s \beta}=0.005 \times 10^{9} / \mathrm{L}-0.001 \times 10^{9} / \mathrm{L}=0.004 \times 10^{9} / \mathrm{L}$. Because the $\mathrm{LoB}$ of the $\mathrm{WBC}=0 \times 10^{9} / \mathrm{L}$, the $\mathrm{LoD}$ of the $\mathrm{WBC}=$
$\mathrm{LoB}+\mathrm{D}_{\mathrm{s} \beta}=0.004 \times 10^{9} / \mathrm{L}$

The median LoD of RBC-BF was calculated by the following formula: $(M)=$ the value of the $30.5 \mathrm{rank}$ $=0.0055 \times 10^{12} / \mathrm{L}$; meanwhile, the 5 th percentile was calculated as follows: 5 th percentile $=60 \times 0.05+0.5=$ the value of the $3.5 \mathrm{rank}=0.002 \times 10^{12} / \mathrm{L} . \mathrm{D}_{\mathrm{s} \beta}=0.0055 \times 10^{12} / \mathrm{L}-0.002 \times 10^{12} / \mathrm{L}$ $=0.0035 \times 10^{12} / \mathrm{L}$. Because the LOb of the RBC $=0 \times 10^{12} / \mathrm{L}$, the $\mathrm{LoD}$ of the $\mathrm{RBC}=\mathrm{LoB}+\mathrm{D}_{\mathrm{s \beta}}=0.0035 \times 10^{12} / \mathrm{L} \approx 0.004 \times 10^{12} / \mathrm{L}$. 
Table 7 Detection results of low-concentration RBC-BF samples

\begin{tabular}{lllllll}
\hline $\begin{array}{l}\text { Theoretical } \\
\text { concentration }\left(\times 10^{12} / \mathrm{L}\right)\end{array}$ & 0.001 & 0.002 & 0.004 & 0.006 & 0.008 & 0.010 \\
\hline 1 & 0.003 & 0.002 & 0.005 & 0.006 & 0.008 & 0.009 \\
2 & 0.002 & 0.002 & 0.004 & 0.006 & 0.009 & 0.012 \\
3 & 0.002 & 0.002 & 0.004 & 0.007 & 0.009 & 0.012 \\
4 & 0.002 & 0.002 & 0.005 & 0.007 & 0.009 & 0.012 \\
5 & 0.002 & 0.002 & 0.004 & 0.006 & 0.008 & 0.011 \\
6 & 0.002 & 0.002 & 0.005 & 0.007 & 0.009 & 0.012 \\
7 & 0.002 & 0.002 & 0.005 & 0.006 & 0.009 & 0.010 \\
8 & 0.003 & 0.003 & 0.005 & 0.006 & 0.009 & 0.010 \\
9 & 0.002 & 0.002 & 0.005 & 0.007 & 0.009 & 0.010 \\
10 & 0.002 & 0.002 & 0.005 & 0.007 & 0.009 & 0.011 \\
Mean & 0.002 & 0.002 & 0.005 & 0.007 & 0.009 & 0.011 \\
\hline
\end{tabular}

RBC-BF, red blood cell-body fluid.

\section{Discussion}

With the progress of the medical science and technology, medical laboratory equipment is developing in the direction of automation and standardization, and the traditional method of manual microscopic examination can no longer meet clinical needs. As a consequence, the automatic analysis of body fluids will become increasingly popular. The Medical Laboratory Quality and Competency Accreditation Guidelines (ISO15189:2012) require medical laboratories to verify or confirm the performance of the selected testing procedures before testing patient samples, confirming that they meet the manufacturer's criteria and ensuring that the test results meet the intended use and clinical requirements (10). There are no independent calibrators and few supporting quality control products in the humoral mode of automatic hematology analyzers at present. As a consequence, it is difficult to achieve strict daily quality management. Therefore, it is particularly important to verify the performance of the humoral mode of the automatic analyzer before it is applied to routine tests in clinical practice (11).

The performance verification results of this study show that the background count results of WBC-BF and RBC$\mathrm{BF}$ of the BC-6000PLUS analyzer (Shenzhen Mindray Biomedical Electronics Co., Ltd.) are 0. It can thus provide a good background for humoral samples with a low cell count. In addition, the contamination rates of WBC-BF and $\mathrm{RBC}-\mathrm{BF}$ of the instrument are 0 . This indicates that the likelihood of cross-contamination between the samples is very low. The BC-6000PLUS analyzer has a wide linear range for both WBC-BF and RBC-BF. Moreover, there is a good linear relationship between detection concentration and dilution ratio. The correlation coefficient $R^{2} \geq 0.95$, which is in line with the manufacturer's performance statement. Therefore, the linear range of WBC-BF and $\mathrm{RBC}-\mathrm{BF}$ can be used as the reportable range of the clinical results. It was difficult to verify the inter-batch precision because the Mindray company does not produce the supporting quality control products of the BC-6000PLUS humoral model. Other limitations include unstable conditions such as cell lysis and spontaneous coagulation of humoral samples. As a consequence, only the intra-batch precision parameters of the instrument were verified. The precision results indicated that the intra-batch precision variation coefficients of the $\mathrm{WBC}-\mathrm{BF}$ and $\mathrm{RBC}-\mathrm{BF}$ of the BC-6000PLUS instrument under the humoral mode at different concentration levels are far lower than the manufacturer's standard, and therefore the repeatability of the instrument is good. There is a high correlation between the results under the humoral mode of the BC-6000PLUS analyzer and those of the manual microscopic examination in terms of instrument accuracy evaluation. The data show that the correlation coefficients of WBC-BF, RBC-BF, $\mathrm{MN}$, and $\mathrm{PMN}$ are greater than $90 \%$, with the linear slope ranging from 0.95 to 1.05 . They all meet the manufacturers' 
stated requirements.

Appendix A of the Clinical Humoral Examination Technology of the WS/T662-2020 clearly stipulates that each laboratory should establish the detection of the lower limit of the nucleated cell count and RBC count of the detection system used. In addition, the detection of the lower limit should not be lower than that of the instrument. The instrument is often unable to detect or guarantee the accuracy of low-value results when the cell count of clinical samples is lower than the lower limit of the instrument. As a consequence, it is necessary to recheck the count by manual microscopic examination, which is more common in cerebrospinal fluid specimens, and to establish the lower detection limit of the cell counting under the instrument's humoral model. Because the Mindray company does not provide any statement regarding the lower detection limits of the items under this instrument's humoral mode, our laboratory had to independently determine the $\mathrm{LoB}$ value and $\mathrm{LoD}$ value of the cell counting under humoral mode for the BC-6000PLUS analyzer. The LoB value refers to the maximum measured value that can be observed in the blank sample, and the LoD value refers to the lowest analyte concentration detected from the sample (12). According to our statistical analysis, the LoB values of both WBC-BF and $\mathrm{RBC}-\mathrm{BF}$ of this instrument are 0 . The $\mathrm{LoD}$ value of $\mathrm{WBC}-\mathrm{BF}$ is $0.004 \times 10^{9} / \mathrm{L}$, and the $\mathrm{LoD}$ value of $\mathrm{RBC}-\mathrm{BF}$ is $0.004 \times 10^{12} / \mathrm{L}$. Thus, our laboratory has set the lower limit of the WBC-BF of the BC-6000PLUS analyzer as $0.004 \times / \mathrm{L}$ and the lower limit of the RBC-BF as $0.004 \times 10^{12} / \mathrm{L}$. The laboratory stipulates that manual microscopic examination must be performed to reexamine the samples with low values if the test results of the instrument are lower than the lower limit.

The present study was limited by the fact that the interbatch precision of the instrument could not be verified due to objective conditions. However, Joob et al. (13) reported that the fully automatic hematology analyzer was equipped with an independent humoral detection mode and can obtain highly accurate results even in humoral samples with a small number of cells; meanwhile the Clinical and Laboratory Standards Institute (CLSI) H56-A guideline (14) also points out that according to the College of American Pathologists (CAP), it is no longer necessary to run humoral cell quality control independently if the instrument is used for blood cell analysis. However, the author suggests that it is still necessary for conditional clinical laboratories to establish an internal quality control management system for automatic detection of humoral samples. It is also believed that in the near future, the indoor or inter-room quality control of automatic detection of humoral samples will be popularized as body fluid quality control products proliferate and costs are reduced.

In conclusion, the BC-6000PLUS has an extremely low background count and carrying contamination rate, high precision, good accuracy and linearity, high correlation with manual microscopic examination, and good overall detection performance. It can fully meet the needs of clinical routine detection. However, it is worth noting that manual microscopic examination is still the gold standard of body fluid tests and cannot be completely replaced by the automatic instrument testing. It is necessary to reexamine the humoral samples by the manual microscopic examination in accordance with the requirements of the procedure documents of the department, especially for the samples whose cell count is lower than the detection limit, or when the scatter plot abnormality indicates that there are special tangible components (abnormal tumor cells, bacteria, fungi, parasites, etc.).

\section{Acknowledgments}

Funding: None.

\section{Footnote}

Reporting Checklist: The authors have completed the MDAR reporting checklist. Available at https://dx.doi. org/10.21037/apm-21-1187

Data Sharing Statement: Available at https://dx.doi. org/10.21037/apm-21-1187

Conflicts of Interest: All authors have completed the ICMJE uniform disclosure form (available at https://dx.doi. org/10.21037/apm-21-1187). JW is an employee in Shenzhen Mindray Bio-Medical Electronic Co., Ltd. The other authors have no conflicts of interest to declare.

Ethical Statement: The authors are accountable for all aspects of the work in ensuring that questions related to the accuracy or integrity of any part of the work are appropriately investigated and resolved. All procedures performed in this study involving human participants were in accordance with the Declaration of Helsinki (as revised in 2013). This clinical study is a retrospective study. It only collects the clinical data of patients, does not interfere 
with the treatment plan of patients, and will not bring physiological risks to patients. Researchers will try their best to protect the information provided by patients, do not disclose personal privacy, and exempt informed consent.

Open Access Statement: This is an Open Access article distributed in accordance with the Creative Commons Attribution-NonCommercial-NoDerivs 4.0 International License (CC BY-NC-ND 4.0), which permits the noncommercial replication and distribution of the article with the strict proviso that no changes or edits are made and the original work is properly cited (including links to both the formal publication through the relevant DOI and the license). See: https://creativecommons.org/licenses/by-nc-nd/4.0/.

\section{References}

1. Dixon LR. The complete blood count: physiologic basis and clinical usage. J Perinat Neonatal Nurs 1997;11:1-18.

2. Turgeon ML. Clinical hematology theory and procedures. 3rd ed Philadelphia: Lippincott 1999;277-313.

3. Aguadero V, Cano-Corres R, Berlanga E, et al. Evaluation of biological fluid analysis using the sysmex $\mathrm{XN}$ automatic hematology analyzer. Cytometry B Clin Cytom 2018;94:680-8.

4. Han JI, Jang HJ, Na KJ. Hematologic and serum biochemical reference intervals of the Oriental white stork (Ciconia boyciana) and the application of an automatic hematologic analyzer. J Vet Sci 2016;17:399-405.

5. Cardoso MR, de Souza-Araújo CN, Talarico MCR, et al. Evaluation of Automatic Blood Analyzer as Screening Method in Fetomaternal Hemorrhage. Biomed Res Int 2019;2019:6481654.

6. International Council for Standardization in Haematology,
Writing Group, Briggs C, et al. ICSH guidelines for the evaluation of blood cell analysers including those used for differential leucocyte and reticulocyte counting. Int J Lab Hematol 2014;36:613-27.

7. Bourner G, De la Salle B, George T, et al. ICSH guidelines for the verification and performance of automated cell counters for body fluids. Int J Lab Hematol 2014;36:598-612.

8. Cho YU, You E, Jang S, et al. Validation of reflex testing rules and establishment of a new workflow for body fluid cell analysis using a Sysmex XN-550 automatic hematology analyzer. Int J Lab Hematol 2018;40:258-67.

9. Clinical and Laboratory Standards Institute. Evaluation of detection capability for clinical laboratory measurement procedures: Approved guideline-second edition. Wayne: PA CLSI Document 2012;EP17-A2.

10. Guzel O, Guner EI. ISO 15189 accreditation: Requirements for quality and competence of medical laboratories, experience of a laboratory I. Clin Biochem 2009;42:274-8.

11. Vis JY, Huisman A. Verification and quality control of routine hematology analyzers. Int J Lab Hematol 2016;38 Suppl 1:100-9.

12. Schneider F, Maurer C, Friedberg RC. International Organization for Standardization (ISO) 15189. Ann Lab Med 2017;37:365-70.

13. Joob B, Wiwanitkit V. Automated Hematology Analyzer for Cell Counting in Body Fluid. Clin Lab 2017;63:1041-0.

14. Clinical and Laboratory Standards Institute (CLSI). Body fluid analysis for cellular composition: Approved guideline. CLSI document H56-A. CLSI, Wayne, USA, 2006.

(English Language Editor: J. Gray)

Cite this article as: Long Y, Liu J, Chen F, Luo Y, Zhao C, Wei J, Chen C, Lin D. Evaluation of humoral pattern detection performance of Mindray BC-6000PLUS blood analyzer based on WS/T 662-2020. Ann Palliat Med 2021;10(6):6446-6454. doi: $10.21037 /$ apm-21-1187 